present lull, at any stage in further progress reference may have to be made once more to the so-called 'myothermic' and 'neurothermic' methods, which have so great an advantage in their quickness and sensitivity, and in the fact that they can determine so fundamental a quantity as the total energy without any injury to the experimental object. The usefulness of these methods will probably not end with the jubilee of their revival.

\title{
Therapeutic and other Applications of X-Rays and Gamma-Rays
}

\section{By Dr. G. W. C. KaYe, o.B.E., National Physical Laboratory}

W ITHIN a period of fifteen years prior to the King's accession, Röntgen had discovered the X-rays and J. J. Thomson the electron, Becquerel had discovered radioactivity and the Curies had isolated radium. The new reign was to prove an era of X-ray and radium research no less fruitful than its predecessor. In particular, the X-ray crystal diffraction experiments of Laue in Germany in 1912, followed by those of the Braggs in England, opened up a new vista of research which has left its mark on physics, found diverse and important applications in industry and is beginning to acquire significance in the biological sciences. Röntgen, who had lived to see many of these developments, died in 1923 in his seventy-eighth year, poor in fortune, but consoled by the beneficent services which his discovery had rendered in the War.

The British Army entered the War relying entirely for its X-rays on induction coils and 'gas' tubes, both well-nigh obsolete now. About 1908, Snook developed the high-tension closed-core transformer with rotary-arm rectifier, and in 1913, Coolidge introduced the hot-cathode X-ray tube. Much development has since followed. The $\mathrm{X}$-ray tube of to-day is self-protected and shockproof, and is even claimed to be climate-proof! The complete equipment is earth shielded, permits precise control and is silent in operation. The high-tension transformer works in conjunction with one or other of the rectifying-valve and condenser circuits, which deliver either pulsating or constant high voltage to the X-ray tube. In routine medical radiology, these voltages range from $60 \mathrm{kv}$. to $200 \mathrm{kv}$., but $400 \mathrm{kv}$. and up to $1,000 \mathrm{kv}$. are now to be found in certain therapeutic centres.

In 1910, X-ray diagnosis was fairly well advanced, but radiation therapy was in its infancy. Protection for the worker was rudimentary or non-existent; and many injuries and deaths resulted. Not until 1921 were the British protection recommendations issued by a representative committee, and these, the first in any country, were used as a basis for international agreement four years later at Stockholm. These recommendations, which are revised triennially by an International Protection Commission, have been adopted throughout practically the whole civilised world. They have not only provided effective safeguards against the working dangers of $\mathrm{X}$-rays, but also have contributed, as no other factor has, to the better housing and general well-being of the X-ray and radium worker. Most radiology departments in 1910 were deplorably housed, whereas the light, roomy and well-ventilated departments of to-day are often a source of pride to hospitals.

Another vital step in the progress of radiation therapy was the adoption of the röntgen (r.) as the international unit of X-ray quantity or dose. The röntgen, which is an air-ionisation unit, was the subject of an international intercomparison in 1931 by the national laboratories of the United States, Germany and Great Britain, a very satisfactory measure of agreement resulting. It is probable that the röntgen will be adopted internationally for gamma radiation also, though further measurements on both gamma and high-voltage $\mathrm{X}$-radiation are required.

Radiography has found considerable application in the industries and arts, for example, in the examination for flaws and other defects in metal castings and forgings, welds and assembled components. Wooden aeroplane parts were examined radiographically in the War. Many millions of elinical thermometers under test have been expeditiously scrutinised at the National Physical Laboratory by the use of X-rays during the last ten years. Other industrial applications include the screening of electrical insulators and golf balls during manufacture. The National Gallery and the Courtauld Institute have recently installed $\mathrm{X}$-ray outfits for the examination of pictures and objects of art. Gamma-rays are resorted to in the radiography of metal specimens too thick for $\mathrm{X}$-rays to tackle.

It is, however, in the world of medicine and surgery that $\mathrm{X}$-rays and radium have been turned to account most outstandingly. X-ray diagnosis has improved in the last twenty-five years to an almost spectacular extent, and radiation therapy, though of more recent growth, has now established its claim to an important place in the treatment of malignant disease. The use of radium for eancer treatment was given impetus in Great 
Britain by the formation in 1929 of the National Radium Trust and Commission, which were constituted to distribute and administer some quarter of a million pounds worth of radium which, it will be remembered, was subscribed for as a national thanksgiving for the King's recovery from his illness. The Trust and Commission were given powers by their Charter not only to augment existing supplies of radium for the treatment of the sick, but also to advance existing knowledge of the best methods of rendering such treatment.

I would here mention that in what follows, I have had the advantage of the experienced and authoritative assistance of Dr. Constance Wood, whose responsibility for the medical opinions expressed I gratefully acknowledge.

While it is still the case that the whole of the gastro-intestinal tract and early carcinoma of the breast are best treated by surgery, X-ray and radium therapy is bidding fair to displace the knife in the treatment of certain other forms of cancer. The response of tumours to radiation demands wide study, each type of tumour having a different response, both clinically and histologically. Views on radiation treatment have changed and the single massive dose of $\mathrm{X}$-radiation, formerly advocated at Erlangen, is now replaced by a sequence of smaller doses spread over a period of time.

As to the quality of radiation required, this depends on circumstances. In certain superficial forms of cancer, such as rodent ulcer, almost any type of radiation will produce healing, whether it be low-voltage X-rays (for which high-power close-proximity tubes are now available) or the beta- or gamma-rays of radium. Other types of cancer, such as squamous epithelioma of the skin and lip, are successfully treated by a few milligrams of heavily screened radium, the residual scar frequently being quite invisible.

The real problem of malignant disease lies, however, in the treatment of glandular metastases. Small quantities of radium or low-voltage X-rays are not capable of destroying cancer cells at a depth ; for the deeper lesions and gland areas we turn either to (a) X-rays which, excited by very high and ever-increasing voltages, tend to approach gamma-rays in quality, or to $(b)$ large quantities of radium placed at distances remote from the skin in an attempt to obtain the large depth doses possible with X-rays. Both methods have their advocates. From a financial point of view, Regaud estimates that when the heavy initial cost and low maintenance costs of a large radium unit are balanced against the lower initial cost and higher running costs of a high-voltage $\mathrm{X}$-ray plant, there is little to choose between them at the present price of radium, though the possibility of an induced radioactive element of sufficiently long life to make its use practicable may perhaps be envisaged. Adequate protective arrangements appear to be possible with either radium or highvoltage $\mathrm{X}$-rays.

The early progress of 'teleradium' was slow, and its ultimate value is a matter on which opinions differ, but the growing belief in its efficacy by those who have had experience is reflected in the rapidly increasing number of large radium units being set up in the world. Paris, which has had a 4 gram unit for some eight years, now has an 8 gram unit. Stockholm has a 5 gram unit in addition to one of 3 grams which it has used during the last six years; New York, Chicago, Buffalo and Toronto each has a 4 gram unit. Great Britain has lagged behind somewhat in its recognition of the value of 'bomb' treatment, partly because cases are not referred for radium treat. ment at as early a stage as in many parts of the Continent. There have, however, been 1 gram units at the Cancer and Westminster Hospitals in London since 1929, while the Radium Commission in 1933 allocated 1 gram units to both these hospitals as well as to Middlesex and University College Hospitals. Edinburgh and Leeds now have also 1 gram units. The Radium Beam Therapy Research Board, set up with State support in 1933, is working with a 5 gram unit since 1933.

In X-ray diagnosis, short-exposure radiography of any part of the human body offers no difficulties to the experienced. With improvement in apparatus and technique, less and less dense bodies can be distinguished, until now the soft tissues and even the extent of tumour growths in them can be depicted. Modern fluorescent screens enable digestive movements, heart beats or lung movements to be watched or cinematographed.

The physiology of the stomach and alimentary tract has been transformed. The presence and extent of internal cancerous growths or small ulcers in the stomach can be definitely established by swallowing barium salt preparations which are opaque to X-rays. Extraordinary anomalies, such as the presence of the stomach in the chest, have been so revealed. Much work has been done recently on the injection of opaque fluids into abnormal tracts, the radiographs clearly demonstrating to the surgeon what difficulties and dangers may be encountered in a projected operation. Some organs, for example, the gall bladder and parts of the kidney tract, have the property of concentrating certain administered liquids so that they presently become opaque to $\mathrm{X}$-rays, and thus outline the organs and facilitate the diagnosis of deep internal disease.

The X-rays assist in demonstrating the extent of involvement and the type of disease in tuber- 
culosis of the lungs. In the treatment of tuberculosis, when by the introduction of air into the chest cavity, the lung has been collapsed to allow it to rest and heal, radiography indicates the state of the lung and when it should be collapsed again. Pathological cavities in the substance of the lung are revealed by introducing into the chest lipiodol, which is an oil rendered opaque by iodising it. Radiography is a valuable aid in the study of heart disease. The heart is sometimes unexpectedly shown to be on the right side of the chest. In radiography of the brain, air may be introduced into the cavities of the brain, so locating and outlining in the radiograph the extent of a tumour and consequently indicating where the surgeon must operate.

The fine trabecular structure of the interior of bones is plainly revealed by the X-rays. Difficult fractures are set under the fluorescent screen, thus assisting in producing perfect restoration of the damaged bone. The development of the human embryo is better understood, and radiographs of the unborn child show the centres of the ossification of the bones before birth and are often of invaluable assistance to the obstetrician.

The X-ray localisation of foreign objects in the body is a routine matter in hospitals, which are called upon to deal with an amazing variety of objects, for example, coins and safety-pins swallowed by children, dental plates inadvertently swallowed, nails, screws, safety razors, etc., deliberately swallowed by prisoners and others, and legacies from the War in the shape of buried bullets and shrapnel.

\section{Genetics Since IgIo}

\section{By Prof. J. B. S. Haldane, F.R.s., Professor of Genetics, University College, London}

$\mathrm{T}^{\mathrm{H}}$ HE state of genetical knowledge in 1910 can be learnt from Bateson's "Mendel's Principles of Heredity", of which the first two editions were published in 1909 and 1913. The principles which Mendel had shown to hold for seven contrasted pairs of characters in Pisum had been extended to a very large number of characters in many plant and animal species, including man. The main additions to these principles were the pure line, multiple allelomorphism, partial linkage between genes, epistasy, and one of the four types of sex linkage now known. The latter discovery, along with cytological work, had made the genetics of sex determination fairly clear. At least one case of extranuclear inheritance had been recorded.

Mendel's theory was based on the hypothesis that a (diploid) heterozygote produced two kinds of (haploid) gametes in equal numbers. This has since been proved in cases where the haploid generation has a number of variable characteristics ; notably by $\mathrm{v}$. Wettstein in mosses, or where individual haploids can be bred from, as by Andersson-Kottö in ferns. In such cases it is found that the products of a single meiosis are always exactly two cells carrying one member of an allelomorphic pair and two carrying the other. When several pairs are segregating, four different haploid types may arise, and a further analysis shows that genetic segregation may be associated with either meiotic division.

The cytological basis of genetics was cleared up by Morgan and his colleagues Bridges, Muller and Sturtevant, working on Drosophila melanogaster. They were able to show that the genes were located at different points on the chromosomes. Adequate chromosome maps exist for several species of Drosophila, and, thanks largely to the co-ordinative work of Emerson, for Zea Mays. Less complete maps exist for Lathyrus (Punnett) Pharbitis (Imai) and other plants. By studying the giant chromosomes of the gland cells in Drosophila, Painter has been able to correlate the theoretically derived gene map with visible structure, and Muller and Prokofieva have detected visible changes associated with a difference affecting a single gene. Using chromosomes which were microscopically distinguishable owing to the attachment of sections of other chromosomes to them, Stern found that the visible changes in any individual could be predicted from its genetical constitution. Thus crossing-over is a physical fact as well as an explanation of genetical phenomena.

Similarly, Darlington has shown that there is a quantitative agreement between microscopically visible chiasmata and genetically detectable crossing-over, and has made it probable that these two events, which are really aspects of the same phenomenon, are both due to the relief of strains produced in the chromosomes by coiling. Equally striking is the cytological evidence that abnormal types of linkage are associated with cytologically visible exchanges of parts between different chromosomes (Muller and Altenburg, Hammarlund and Hakansson, Dobzhansky, Mcclintock, etc.).

Oenothera Lamarckiana and other forms which breed nearly, but not quite, true, have been shown to be examples of permanent heterozygosis. Here the organism forms two kinds of gametes, but only one of the three possible combinations is produced, owing to inviability or competition. 